\title{
Real-World Incidence of Suboptimal Response to Anti-Tumor Necrosis Factor Therapy for Ulcerative Colitis: A Nationwide Population-Based Study
}

\author{
Ju-Young Shin ${ }^{1}$, Hye-Min Park ${ }^{1,2}$, Min-Young Lee ${ }^{2}$, Ja-Young Jeon ${ }^{3}$, Hyun-Jeong Yoo ${ }^{3}$, and Byong Duk Ye \\ ${ }^{1}$ School of Pharmacy, Sungkyunkwan University, ${ }^{2}$ VIAplus, Suwon, ${ }^{3}$ Pfizer Pharmaceuticals Korea Ltd., and ${ }^{4}$ Department of \\ Gastroenterology and Inflammatory Bowel Disease Center, Asan Medical Center, University of Ulsan College of Medicine, Seoul, Korea
}

See editorial on page 793.

\section{Article Info}

Received November 23, 2020

Revised January 2, 2021

Accepted January 8, 2021

\section{Corresponding Author}

Byong Duk Ye

ORCID https://orcid.org/0000-0001-6647-6325

E-mail bdye@amc.seoul.kr
Background/Aims: Although anti-tumor necrosis factor (TNF) agents have been widely used to treat ulcerative colitis (UC), the real-world incidence of suboptimal response to anti-TNF agents has not been thoroughly investigated, especially among Asians.

Methods: Using the Korean National Health Insurance database, we collected data on UC patients who initiated anti-TNF agents between July 1, 2014, and June 30, 2017. We assessed suboptimal responses, including anti-TNF discontinuation or dose escalation, switching to other biologics, augmentation with a non-biologic therapy, and the requirement for colectomy.

Results: A total of 1,268 patients were included as new anti-TNF users (infliximab 713, adalimumab 433, golimumab 122). The proportion of patients who experienced at least one suboptimal response within 1 year among all patients was $63.5 \%$, including $59.1 \%, 69.5 \%$, and $68.0 \%$ of patients treated with infliximab, adalimumab, and golimumab, respectively. The cumulative incidences of at least one suboptimal response over time were $41.5 \%, 63.7 \%, 80.5 \%$, and $87.1 \%$ at $6,12,24$, and 36 months, respectively. Cox proportional hazards modeling revealed that adalimumab was associated with a higher risk of at least one suboptimal response (hazard ratio [HR], 1.29; $95 \%$ confidence interval [Cl], 1.13 to 1.48), dose escalation ( $\mathrm{HR}, 4.35 ; 95 \% \mathrm{Cl}, 2.97$ to 6.38 ) and discontinuation ( $\mathrm{HR}, 1.25 ; 95 \% \mathrm{Cl}, 1.03$ to 1.52$)$ than infliximab. Golimumab was associated with a higher risk of switching to other biologics than infliximab ( $\mathrm{HR}, 1.78 ; 95 \% \mathrm{Cl}, 1.21$ to 2.60 ).

Conclusions: More than half of Korean UC patients had suboptimal responses to anti-TNF agents within 1 year. UC patients treated with infliximab might be less prone to suboptimal responses than those treated with adalimumab or golimumab. (Gut Liver 2021;15:867-877))

Key Words: Ulcerative colitis; Tumor necrosis factor Inhibitors; Suboptimal response

\section{INTRODUCTION}

Ulcerative colitis (UC) is characterized by waxing and waning chronic inflammation involving the colon, and it is progressive in a substantial proportion of patients. ${ }^{1,2}$ The incidence and prevalence of UC have steadily increased among non-Caucasians, including Asians. ${ }^{3-5}$ In Korea, the age- and sex-adjusted mean annual incidence rate increased almost 20-fold, from $0.33 / 100,000$ in 1986 to $6.58 / 100,000$ in $2015,{ }^{6}$ and the standardized prevalence also increased from $41.41 / 100,000$ in 2009 to $65.95 / 100,000$ in $2016 .^{7}$
Anti-tumor necrosis factor (TNF) agents have revolutionized UC therapy and are being increasingly used for patients with moderate to severe UC., With their increasing use, anti-TNF agents have been reported to be key drivers of UC treatment costs in Europe and East Asia, including Korea. ${ }^{9-13}$ Therefore, the optimized use of antiTNFs has become crucial for mitigating this economic burden and for the proper allocation of UC management resources.

Despite the effectiveness of anti-TNF therapies, not all patients respond to anti-TNFs, and the risk of adverse events, such as infection or malignancy, has not been com- 
pletely resolved. ${ }^{14,15}$ Inadequate responses to, or adverse events associated with, anti-TNFs might necessitate stopping anti-TNFs, switching to other biologics or small molecules, augmenting non-biologic drugs, escalating the antiTNF dose, or colectomy. Changes in anti-TNF treatment patterns and the requirement for colectomy are indicators of suboptimal responses of UC to anti-TNF therapies.

Suboptimal response indicators could be utilized to estimate the effective and safe use of biologics in the realworld. ${ }^{16-20}$ However, only limited data are available on suboptimal responses to anti-TNF therapy among UC patients in Asia, especially in the era of biologics other than nonanti-TNFs. ${ }^{21,22}$ We aimed to identify suboptimal responses to anti-TNF therapies and differences between anti-TNF agents for UC patients in Korea using nationwide population-based data.

\section{MATERIALS AND METHODS}

\section{Data source}

The National Health Insurance database from the Health Insurance Review and Assessment Service (HIRA) in Korea contains approximately 50 million patients, which accounts for $98 \%$ of the entire population. The database includes claims from almost 80,000 healthcare providers across Korea. Analyses of this database can be generalized to the entire Korean population. ${ }^{23}$

The study protocol was approved by the Institutional Review Boards of Asan Medical Center (IRB number: 2019-1251) and Sungkyunkwan University (IRB number: SKKU 2019-03-009), who waived the requirement for informed consent from study participants.

\section{Study design and subjects}

We conducted a retrospective cohort study using HIRA data from July 1, 2013, to June 30, 2018. Our study subjects were UC patients who started anti-TNF therapy between July 1, 2014, and June 30, 2017 and did not take any biologic therapies or targeted biologic therapies for other diagnoses within the previous year. The follow-up starting date in this study - the index date-was defined as the first date when biologic therapy was prescribed. All patients were required to have data available for a minimum of 12 months before the index date (baseline period) and 12 months after the index date (follow-up period) (Fig. 1).

Patients were eligible if they met all of the following criteria: at least one claim involving the K51.x International Classification of Diseases, 10th revision (ICD-10) code for UC during the study period, at least one claim for targeted biologic therapy during the index period, a claim with an appropriate diagnosis code for UC at the index date (the K51.x ICD-10 code and the V131 rare/intractable diseases patient support program registration code denote UC), and age $\geq 18$ years at the index date. Patients were excluded if they satisfied any of the following: had a claim for any biologic therapies or tofacitinib (this criterion was to check whether or not biologic therapies had been used for new users only) during the baseline period, or had a claim with a diagnostic code for other indications of targeted biologic therapies (Supplementary Table 1) during the baseline period.

\section{Infliximab, adalimumab, and golimumab cohorts}

Study subjects were classified into infliximab, adalimumab, and golimumab cohorts according to index antiTNF therapies. As index treatments were assorted according to active substances, the infliximab cohort included patients who used infliximab biosimilars. For patients to be eligible, index treatments needed to be prescribed for $\mathrm{UC}$ and be reimbursed within the index period.

\section{Study variables}

1) Baseline characteristics

We captured data on patient sex, age, Charlson comorbidity index, insurance type, medical facility type, and prior/concomitant treatments. Age, insurance type, and types of medical facilities were defined at the initiation of biologic therapy. Charlson comorbidity index scores were calculated with disease codes captured during the baseline period. ${ }^{24}$ Prior treatments were defined as conventional therapies prescribed within 90 days before the initiation of biologic therapy. Concomitant treatment was defined as

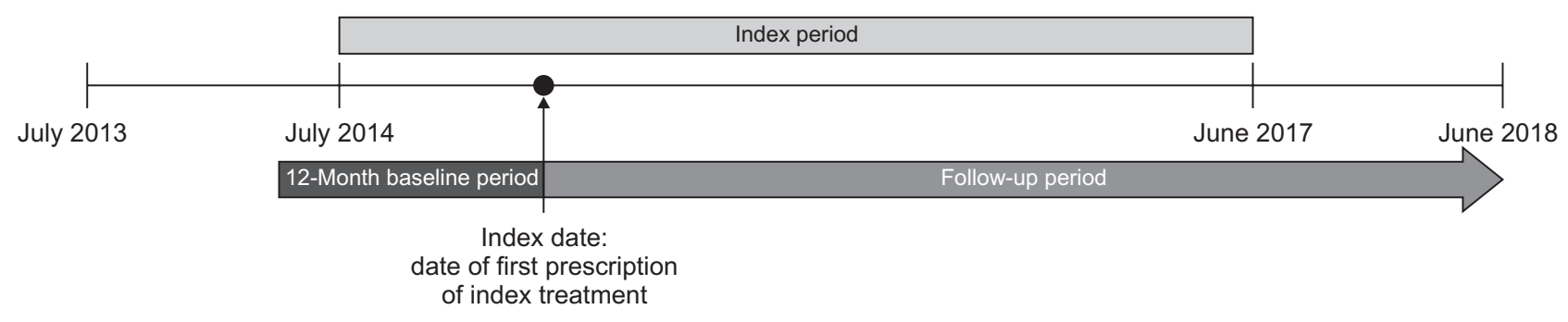

Fig. 1. Study design scheme. 
conventional therapies (5-aminosalicylic acids [5-ASA], corticosteroids, immunomodulators) prescribed within 60 days after the initiation of biologic therapies. Types of conventional therapies were subdivided by formulations (systemic or topical for 5-ASAs/corticosteroids) or ingredients (thiopurines, calcineurin inhibitors, or methotrexate for immunomodulators).

\section{2) Suboptimal response}

Indicators of suboptimal responses included discontinuation of anti-TNF agents, switching to other biologics, augmentation, dose escalation of anti-TNF, and colectomy. At least one suboptimal treatment was identified based on the suboptimal event that first occurred. Discontinuation of anti-TNF was defined as a gap of $\geq 90$ days between the expected last day of supply of an anti-TNF prescription and the next prescription, with no prescriptions of any biologic therapies during the gap. The date of discontinuation was defined as the last day of supply of the index treatment prescription just before the gap. Switching was defined as a transition from the index treatment to another biologic therapy (switched treatment) within 90 days after the prescription of the index treatment. The switching date was the date of the first prescription of the switched treatment. Augmentation was defined as an addition of conventional therapies. To differentiate from concomitant treatment with anti-TNF agents, we observed augmentation from the end of the observation period for concomitant treatments (within "index date+60 days"). Additionally, to be considered augmentation, the prescription of conventional medication for more than 28 days, together with index treatment, was required. Augmented treatments were also required to have not been prescribed within 90 days preceding the date of adding conventional drugs. The addition of conventional drugs of the same type with concomitant drugs (i.e., augmentation of topical 5-ASA in a case already under topical 5-ASA as concomitant treatment) was not considered augmentation. The date of augmentation was defined as the date when the added conventional therapies were first prescribed. Anti-TNF dose escalation was defined as a dose increase during the maintenance phase resulting in doubling the dose (in terms of amount or frequency) relative to the recommended standard maintenance dose for UC. The maintenance phase depended on the index treatments (infliximab: after three doses within 14 weeks; adalimumab: after five doses with 10 weeks; golimumab: after four doses within 14 weeks). The date of dose escalation was the date of first prescription of the increased anti-TNF dose. Colectomy was defined as the performance of colonic resectional surgery for UC (Supplementary Table 2). Colectomy date was the date of the first UC-related operation.

\section{3) Follow-up}

The study patients were followed until the last day of observation (June 30,2018) or the development of a suboptimal response.

\section{Statistical analysis}

Baseline characteristics are expressed as numbers with percentages, or means with standard deviations. Also, we summarized the occurrence of suboptimal responses during the first year after initiating biologic therapy as the number of patients and percentage. The chi-square or analysis of variance tests were conducted for comparing the differences in baseline characteristics and suboptimal responses during the first year among the three cohorts. Time to occurrence of a suboptimal response was expressed as median with interquartile range (IQR). The cumulative suboptimal response incidence at $6,12,18,24,30$, and 36 months was estimated using the Kaplan-Meier method, and times to occurrences of suboptimal responses were compared between cohorts using the log-rank test. To estimate the hazard ratios (HRs) of each suboptimal event among users of adalimumab and golimumab, compared with those of infliximab, the Cox proportional hazards models were used, with adjustments for covariates, including age, sex, Charlson comorbidity index score, insurance type, types of medical facilities, use of prior treatments by each type (5-ASA, corticosteroids, immunomodulators), and use of concomitant treatments by each type (5-ASA, corticosteroids, immunomodulators). All analyses were performed using SAS 9.4 software (SAS Institute, Cary, NC, USA), and p-values $<0.05$ was deemed to be statistically significant.

\section{RESULTS}

\section{Baseline characteristics}

A total of 1,268 patients were identified through patient selection criteria, and patients were allocated to infliximab cohort $(n=713)$, adalimumab cohort $(n=433)$, and golimumab cohort ( $\mathrm{n}=122$ ) (Fig. 2). Baseline characteristics are summarized in Table 1. Patients in the infliximab cohort were more frequently prescribed anti-TNF agents at tertiary hospitals. Over $90 \%$ of the patients had been previously treated with 5-ASA. Corticosteroids were previously given to $77.4 \%$ of patients and immunomodulators to $52.8 \%$. The proportions of patients who concomitantly used 5-ASA, corticosteroids, and immunomodulators were $90.3 \%, 55.1 \%$, and $55.1 \%$, respectively. 


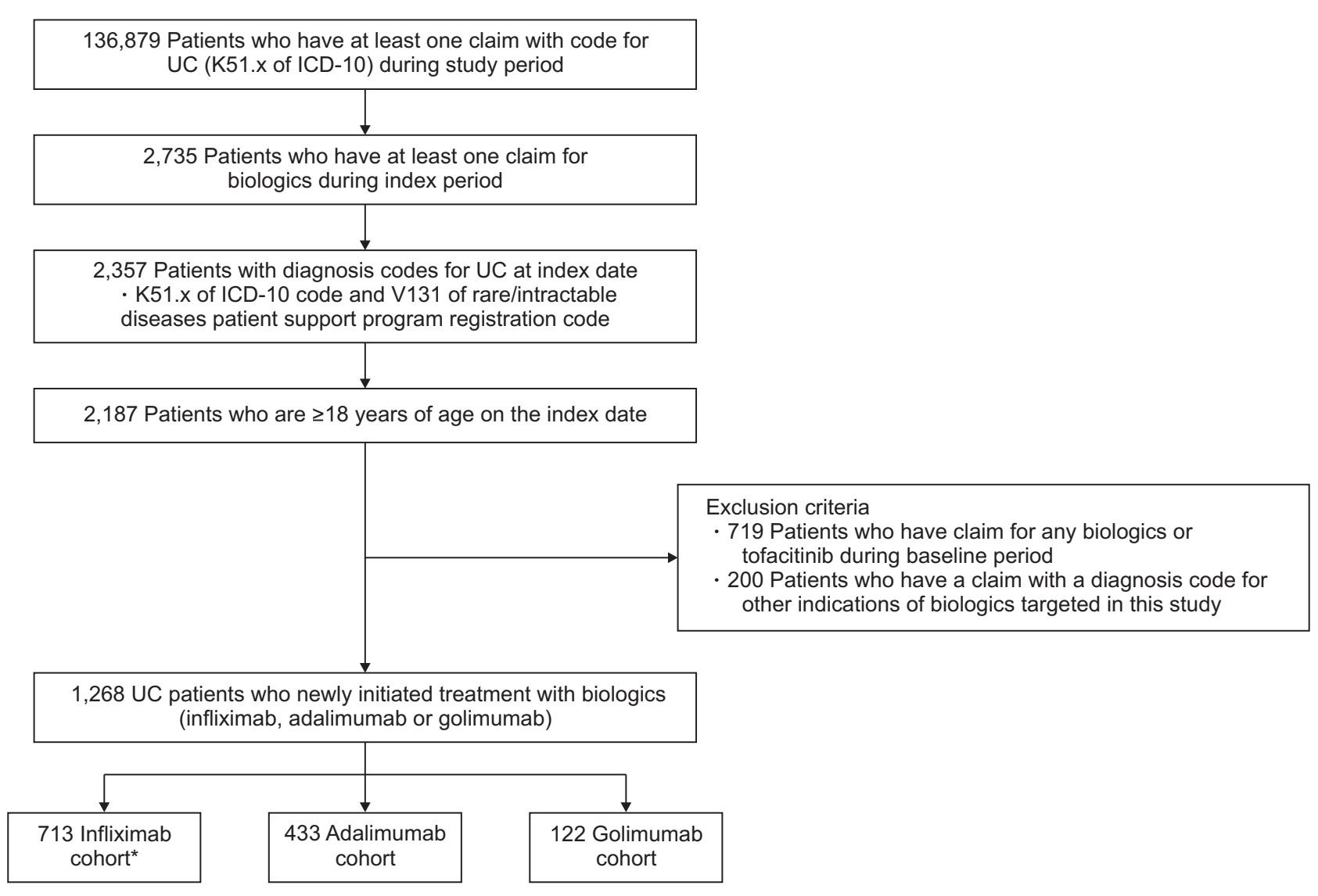

Fig. 2. Patient selection.

UC, ulcerative colitis; ICD-10, International Classification of Diseases, 10th revision. *Since index treatments were extracted based on active substances, the infliximab cohort included patients who used infliximab biosimilars.

\section{Suboptimal response during the first year after initiating anti-TNF therapy}

During the first year after initiating anti-TNF therapy, at least one suboptimal response to anti-TNF agents occurred in 805 patients $(63.5 \%)$. The most common subtype of suboptimal response indicator was augmentation of conventional therapies (25.3\%), followed by discontinuation of anti-TNFs (25.0\%). Colectomy was required in only 12 patients (1.0\%). The proportion of patients experiencing at least one suboptimal response to infliximab was lower than that for the other agents (infliximab, 59.1\%; adalimumab, 69.5\%; golimumab, 68.0\%; $\mathrm{p}<0.001)$. Switching from index treatment to another biologic treatment tended to occur more frequently in the golimumab cohort $(\mathrm{p}=0.015)$. Corticosteroids were the most frequently prescribed agents for non-biologic augmentation (46.4\%). Dose escalation was performed in $16.2 \%$ of the adalimumab cohort, which was a significantly higher proportion than the other two cohorts $(\mathrm{p}<0.001)$ (Table 2).

\section{Cumulative incidences of suboptimal responses}

The cumulative incidences of suboptimal responses were $41.5 \%, 63.7 \%, 74.3 \%, 80.5 \%, 84.8 \%$, and $87.1 \%$ at 6 , $12,18,24,30$, and 36 months, respectively. The cumulative incidences of suboptimal response and subtypes of suboptimal response according to cohort over time are presented in Fig. 3. The median time to suboptimal response was 241 days (IQR, 104 to 468 days) (infliximab cohort: 280 days [IQR, 106 to 520 days]; adalimumab cohort: 213 days [IQR, 99 to 418 days]; golimumab cohort: 199 days [IQR, 97 to 407 days]), and the time to suboptimal response was significantly different among the cohorts $(\mathrm{p}=0.003)$. The time to suboptimal response was significantly longer in the infliximab cohort compared with the adalimumab cohort $(p<0.001)$, but not different between the infliximab and golimumab cohorts $(\mathrm{p}=0.151)$ or between the adalimumab and golimumab cohorts $(\mathrm{p}=0.674)$.

\section{Risk of suboptimal response by index treatment}

The multivariable Cox proportional hazard model to compare the suboptimal response risks revealed that the adalimumab cohort was significantly more likely to experience at least one suboptimal response than the infliximab cohort (HR, 1.29; 95\% confidence interval [CI], 1.13 to 
Table 1. Baseline Characteristics of Ulcerative Colitis Patients Newly Initiated on Biologic Therapies

\begin{tabular}{|c|c|c|c|c|c|}
\hline \multirow{2}{*}{ Characteristics } & \multirow{2}{*}{$\begin{array}{l}\text { All patients } \\
(n=1,268)\end{array}$} & \multicolumn{4}{|c|}{ By cohort } \\
\hline & & Infliximab (n=713) & Adalimumab (n=433) & Golimumab (n=122) & $\mathrm{p}$-value \\
\hline Female sex & 472 (37.2) & $274(38.4)$ & $159(36.7)$ & 39 (32.0) & 0.380 \\
\hline Age, yr & $43.55 \pm 15.30$ & $43.59 \pm 15.30$ & $43.72 \pm 15.40$ & $42.70 \pm 14.90$ & 0.803 \\
\hline $18-29$ & $287(22.6)$ & $161(22.6)$ & $95(21.9)$ & $31(25.4)$ & 0.961 \\
\hline $30-39$ & $230(18.1)$ & $129(18.1)$ & 79 (18.2) & $22(18.0)$ & \\
\hline $40-49$ & $284(22.4)$ & $158(22.12)$ & $101(23.3)$ & $25(20.5)$ & \\
\hline $50-64$ & 346 (27.3) & $193(27.1)$ & $117(27.0)$ & $36(29.5)$ & \\
\hline$\geq 65$ & $121(9.5)$ & $72(10.1)$ & $41(9.5)$ & $8(6.6)$ & \\
\hline $\mathrm{CCl}$ & $1.19 \pm 1.30$ & $1.17 \pm 1.30$ & $1.23 \pm 1.40$ & $1.16 \pm 1.10$ & 0.737 \\
\hline 0 & $460(36.3)$ & $261(36.6)$ & 157 (37.3) & $42(34.4)$ & 0.991 \\
\hline 1 & $415(32.7)$ & $233(32.7)$ & 142 (32.8) & 40 (32.8) & \\
\hline$\geq 2$ & $393(31.0)$ & $219(30.7)$ & $134(31.0)$ & $40(32.8)$ & \\
\hline Insurance types & & & & & 0.596 \\
\hline National Health Insurance & $1,242(98.0)$ & $696(97.6)$ & 425 (98.2) & 121 (99.2) & \\
\hline Medical aid & $26(2.1)$ & $17(2.4)$ & $8[1.9]$ & $1(0.8)$ & \\
\hline Types of medical institution & & & & & $<0.001$ \\
\hline Tertiary hospitals & 783 (61.8) & 480 (67.3) & $236(54.5)$ & $67(54.9)$ & \\
\hline General hospitals & 395 (31.2) & $186(26.1)$ & $178(41.1)$ & $31(25.4)$ & \\
\hline Hospitals & $71(5.6)$ & $34(4.8)$ & $16(3.70)$ & $21(17.2)$ & \\
\hline Clinics & $19(1.5)$ & $13(1.8)$ & $3(0.7)$ & $3(2.5)$ & \\
\hline \multicolumn{6}{|l|}{ Prior treatments* } \\
\hline None & $36(2.8)$ & $21(3.0)$ & $11(2.5)$ & 4 (3.3) & 0.880 \\
\hline At least 1 unique type of treatment & $1,232(97.2)$ & $692(97.1)$ & 422 (97.5) & $118(96.7)$ & \\
\hline With 1 unique type of treatment & $140(11.0)$ & $85(11.9)$ & $21(9.5)$ & $14(11.5)$ & \\
\hline With 2 unique types of treatment & $590(46.5)$ & $317(44.5)$ & $216(49.9)$ & $57(46.7)$ & \\
\hline With 3 unique types of treatment & $502(39.6)$ & $290(40.7)$ & $165(38.1)$ & $47(38.5)$ & \\
\hline \multicolumn{6}{|l|}{ Use of prior treatments by type* } \\
\hline 5-ASA & $1,175(92.7)$ & $649(91.0)$ & 409 (94.5) & 117 (95.9) & 0.034 \\
\hline Oral & 1,140 (89.9) & $631(88.5)$ & 395 (91.2) & $114(93.4)$ & 0.131 \\
\hline Topical & $655(51.7)$ & $353(49.5)$ & $231(53.4)$ & 71 (58.2) & 0.142 \\
\hline Corticosteroids & $981(77.4)$ & $560(78.5)$ & $334(77.1)$ & 87 (71.3) & 0.209 \\
\hline Systemic & $960(75.7)$ & $552(77.4)$ & $323(74.6)$ & $85(69.7)$ & 0.146 \\
\hline Topical & $172(13.6)$ & 95 (13.3) & $58(13.4)$ & $19(15.6)$ & 0.792 \\
\hline Immunomodulators & $670(52.8)$ & $380(53.5)$ & $225(52.0)$ & 65 (53.3) & 0.904 \\
\hline Thiopurine & $667(52.6)$ & 379 (53.2) & 223 (51.5) & 65 (53.3) & 0.852 \\
\hline Calcineurine inhibitors & $3(0.2)$ & $1(0.1)$ & $2(0.5)$ & 0 & $0.676^{+}$ \\
\hline Methotrexate & $1(0.1)$ & 0 & $1(0.2)$ & 0 & $0.438^{+}$ \\
\hline \multicolumn{6}{|l|}{ Concomitant treatments* } \\
\hline None & $38(3.0)$ & $19(2.7)$ & $17(3.9)$ & $2(1.6)$ & 0.312 \\
\hline At least 1 unique type of treatment & $1,230(97.0)$ & 694 (97.3) & $416(96.1)$ & $120(98.4)$ & \\
\hline With 1 unique type of treatment & $279(22.0)$ & $163(22.9)$ & 91 (21.0) & $25(20.5)$ & \\
\hline With 2 unique types of treatment & $589(46.5)$ & $340(47.7)$ & $186(43.0)$ & $63(51.6)$ & \\
\hline With 3 unique types of treatment & $362(28.6)$ & $191(26.8)$ & $139(32.1)$ & $32(26.2)$ & \\
\hline \multicolumn{6}{|l|}{ Use of concomitant treatments by type* } \\
\hline 5-ASA & $1,145(90.3)$ & $641(90.0)$ & 391 (90.3) & $113(92.6)$ & 0.644 \\
\hline Oral & $1,115(87.9)$ & $623(87.4)$ & $384(88.7)$ & 108 (88.5) & 0.788 \\
\hline Topical & 475 (37.5) & $254(35.6)$ & $169(39.0)$ & $52(42.6)$ & 0.238 \\
\hline Corticosteroids & $699(55.1)$ & $352(49.4)$ & $272(62.8)$ & $75(61.5)$ & $<0.001$ \\
\hline Systemic & $657(51.8)$ & $327(45.9)$ & $259(59.8)$ & 71 (58.2) & $<0.001$ \\
\hline Topical & $111(8.8)$ & $60(8.4)$ & $43(9.9)$ & $8(6.6)$ & 0.452 \\
\hline Immunomodulators & $699(55.1)$ & 423 (59.3) & $217(50.1)$ & $59(48.4)$ & 0.003 \\
\hline Thiopurine & $691(54.5)$ & $419(58.8)$ & $213(49.2)$ & $59(48.4)$ & 0.003 \\
\hline Calcineurine inhibitors & 4 (0.3) & $2(0.3)$ & 2 (0.5) & 0 & $0.757^{+}$ \\
\hline Methotrexate & $7(0.6)$ & $4(0.6)$ & $2(0.5)$ & $1(0.8)$ & $0.773^{+}$ \\
\hline
\end{tabular}

Data are presented as number $(\%)$ or mean \pm SD. p-values were calculated with the chi-square or ANOVA tests depending on whether the variables were continuous or categorical.

$\mathrm{CCl}$, Charlson comorbidity index; 5-ASA, 5-aminosalicylic acid; ANOVA; analysis of variance.

*Including 5-ASA, corticosteroids, immunomodulators as types, not subdividing by formulation or detailed ingredients; ${ }^{\dagger}$ Fisher exact test. 
Table 2. Suboptimal Response Indicators within 1 Year after Initiating Biologic Therapies in Ulcerative Colitis Patients

\begin{tabular}{|c|c|c|c|c|c|}
\hline \multirow[b]{2}{*}{ Indicator } & \multirow{2}{*}{$\begin{array}{c}\text { All patients } \\
(n=1,268)\end{array}$} & \multicolumn{4}{|c|}{ By cohort } \\
\hline & & $\begin{array}{c}\text { Infliximab } \\
\text { (n=713) }\end{array}$ & $\begin{array}{l}\text { Adalimumab } \\
(\mathrm{n}=433)\end{array}$ & $\begin{array}{l}\text { Golimumab } \\
\text { (n=122) }\end{array}$ & $p$-value \\
\hline At least 1 suboptimal measures & $805(63.5)$ & $421(59.1)$ & 301 (69.5) & $83(68.0)$ & $<0.001$ \\
\hline Discontinuation & $317(25.0)$ & 176 (24.7) & 109 (25.2) & 32 (26.2) & 0.931 \\
\hline Switching & $208(16.4)$ & $114(16.0)$ & $63(14.6)$ & $31(25.4)$ & 0.015 \\
\hline to infliximab & $68(32.7)$ & 0 & 48 (76.2) & 20 (64.5) & \\
\hline to adalimumab & $110(52.9)$ & 99 (86.8) & 0 & $11(35.5)$ & \\
\hline to golimumab & $30(14.4)$ & 15 (13.2) & 15 (23.8) & 0 & \\
\hline Augmentation & $321(25.3)$ & $179(25.1)$ & $117(27.0)$ & $25(20.5)$ & 0.336 \\
\hline 5-ASA & $34(10.6)$ & $16(8.9)$ & 12 (10.3) & $6(24.0)$ & \\
\hline Oral & $2(0.6)$ & 0 & $1(0.9)$ & $1(4.0)$ & \\
\hline Topical & $32(12.0)$ & $16(8.9)$ & $11(9.4)$ & $5(20.0)$ & \\
\hline Corticosteroids & $149(46.4)$ & 81 (45.3) & $57(48.7)$ & $11(44.0)$ & \\
\hline Systemic & $118(36.8)$ & $69(38.6)$ & 40 (34.2) & $9(36.0)$ & \\
\hline Topical & $31(9.7)$ & $12(6.7)$ & $17(14.5)$ & $2(8.0)$ & \\
\hline Immunomodulators & $138(43.0)$ & $82(45.8)$ & $48(41.0)$ & $8(32.0)$ & \\
\hline Thiopurine & $134(41.7)$ & $79(44.1)$ & $47(40.2)$ & $8(32.0)$ & \\
\hline Calcineurine inhibitors & $1(0.3)$ & $1(0.6)$ & 0 & 0 & \\
\hline Methotrexate & $3(0.9)$ & $2(1.1)$ & $1(0.9)$ & 0 & \\
\hline Dose escalation & $102(8.0)$ & $27(3.8)$ & 70 (16.2) & $5(4.1)$ & $<0.001$ \\
\hline Colectomy & $12(1.0)$ & $7(0.9)$ & $4(0.9)$ & $1(0.8)$ & $>0.999^{*}$ \\
\hline
\end{tabular}

Data are presented as number (\%).

5-ASA, 5-aminosalicylic acid.

*Fisher exact test.

1.48). Regarding subtypes of suboptimal response indicators, the adalimumab cohort had a significantly higher risk of drug discontinuation (HR, 1.25; 95\% CI, 1.03 to 1.52 ) and dose escalation (HR, 4.35; 95\% CI, 2.97 to 6.38) compared with the infliximab cohort. On the other hand, the golimumab cohort had a significantly higher risk than the infliximab cohort of switching to other biologics (HR, 1.78; 95\% CI, 1.21 to 2.60 ) (Table 3).

\section{DISCUSSION}

Our nationwide population-based study found that approximately two-thirds of UC patients experienced at least one suboptimal response within the first year of initiating anti-TNF therapies and that the proportion of patients with at least one suboptimal response steadily increased over time, reaching $90 \%$ at 36 months. The main subtypes of suboptimal response indicators were augmentation with conventional therapies and discontinuation of anti-TNF agents. Infliximab was associated with a lower suboptimal response risk than adalimumab or golimumab.

The risk of suboptimal response to anti-TNFs in our study appears to be largely similar to findings from the Western world. ${ }^{17,18}$ A previous study using a U.S. commercial claims database from 2005 and 2013 showed that $72 \%$ and $91 \%$ of patients with UC experienced at least one suboptimal response after 12 and 36 months, respectively; these figures were numerically slightly higher than those observed in our study. ${ }^{17}$ In contrast, a multinational study using UC patient data from Canada, France, Germany, Italy, Spain, and the United Kingdom reported that $64.1 \%$ of anti-TNF initiators experienced at least one suboptimal response within 2 years, which was lower than the frequencies from the U.S. study and those from our study. ${ }^{17,18}$ However, disease-related urgent care was included as a suboptimal response indicator, and augmentation included a wider range of drugs, such as antibiotics, likely contributing to the higher rates of suboptimal responses in the U.S. study compared with ours. ${ }^{17}$ Different study populations as well as differences in the study periods; and definitions of discontinuation, augmentation, and practice pattern might also have contributed to the differing results. ${ }^{17,18}$ A previous Korean study utilizing the Korean National Health Insurance claims database reported that among 807 UC patients who initiated anti-TNFs between 2010 and 2012, 72.6\% experienced non-persistence of the drugs. ${ }^{21}$ Despite some numerical differences in the incidences of suboptimal therapy with anti-TNF agents, the present study and previous studies are generally consistent in that more than half of UC patients experience suboptimal responses after initiating biologic therapies, highlighting the unmet need in the treatment of patients with moderate to severe UC. ${ }^{17,18,21}$

The frequencies of indicators of suboptimal anti-TNF 


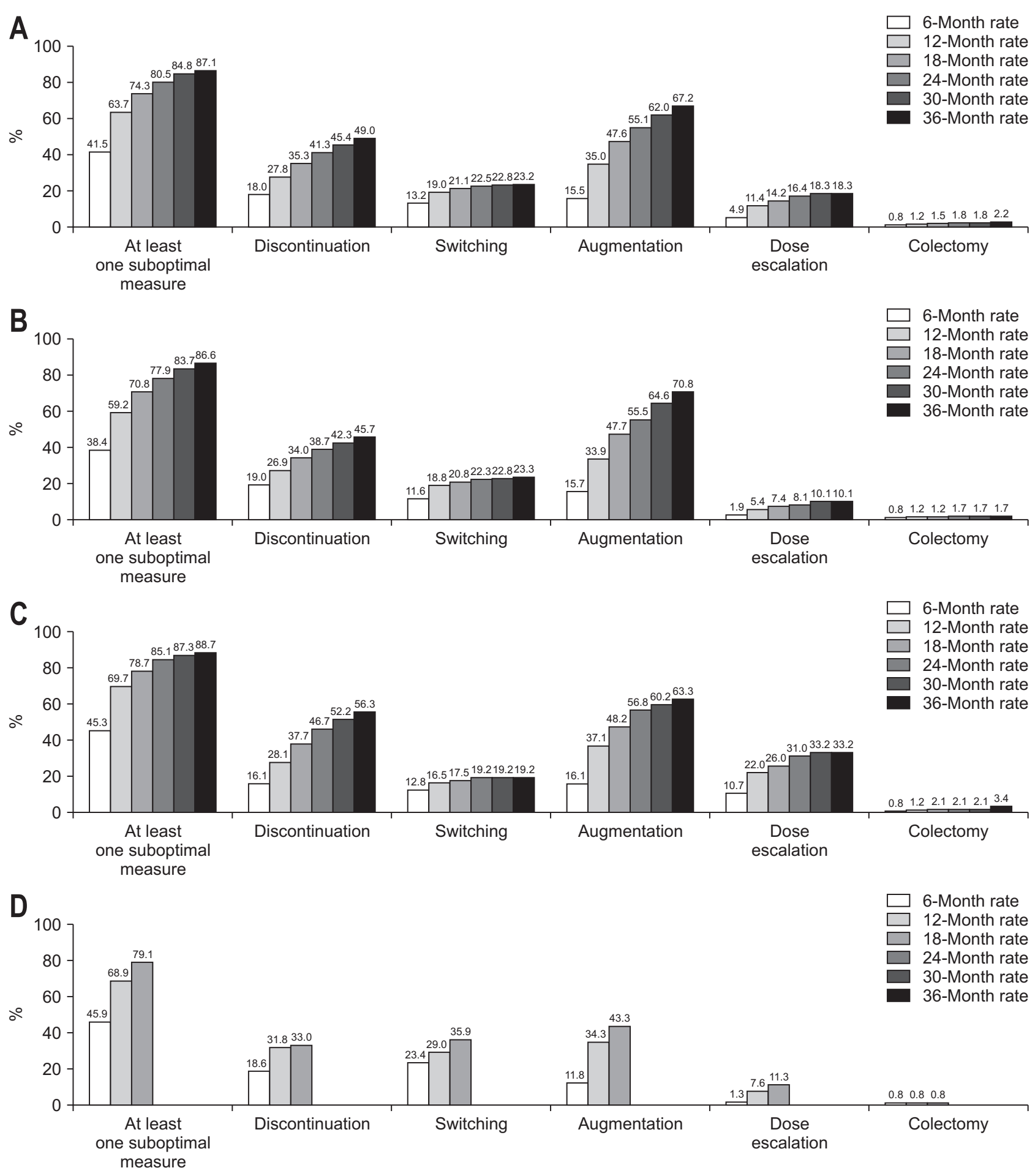

Fig. 3. Cumulative incidences of suboptimal responses by cohorts. (A) Cumulative incidence of suboptimal responses in all cohorts. (B) Cumulative incidence of suboptimal responses in infliximab cohort. (C) Cumulative incidence of suboptimal responses in adalimumab cohort. (D) Cumulative incidence of suboptimal responses in golimumab cohort. Since no more suboptimal events in the golimumab cohort were detected after 18 months, the figures for the golimumab cohort are presented only until 18 months.

therapy were similar between previous Western studies and our study, except that fewer patients in our study experienced anti-TNF dose escalation. ${ }^{17,18}$ The limited reimbursement for infliximab and golimumab dose escala- tion in contrast with adalimumab for UC in Korea might have been responsible for the low rates of dose escalation. However, even for adalimumab, dose escalation occurred for less than $20 \%$ of patients. Insufficient awareness of the 
Table 3. Cox Proportional Hazards Regression for the Risk of Each Suboptimal Response Indicator among the Biologic Therapies

\begin{tabular}{|c|c|c|c|c|}
\hline \multirow{2}{*}{ Suboptimal response indicator } & \multicolumn{2}{|c|}{ Univariable analysis } & \multicolumn{2}{|c|}{ Multivariable analysis* } \\
\hline & Hazard ratio $(95 \% \mathrm{Cl})$ & p-value & Hazard ratio $(95 \% \mathrm{Cl})$ & p-value \\
\hline \multicolumn{5}{|l|}{ At least 1 suboptimal response } \\
\hline Infliximab & Reference & & Reference & \\
\hline Adalimumab & $1.25(1.10-1.43)$ & $<0.001$ & $1.29(1.13-1.48)$ & $<0.001$ \\
\hline Golimumab & $1.18(0.95-1.47)$ & 0.139 & $1.19(0.95-1.49)$ & 0.130 \\
\hline \multicolumn{5}{|l|}{ Discontinuation } \\
\hline Infliximab & Reference & & Reference & \\
\hline Adalimumab & $1.28(1.06-1.54)$ & 0.012 & $1.25(1.03-1.52)$ & 0.023 \\
\hline Golimumab & $0.97(0.67-1.39)$ & 0.848 & $0.89(0.61-1.30)$ & 0.546 \\
\hline Switching & & & & \\
\hline Infliximab & Reference & & Reference & \\
\hline Adalimumab & $0.88(0.65-1.17)$ & 0.372 & $0.81(0.60-1.09)$ & 0.169 \\
\hline Golimumab & $1.84(1.27-2.66)$ & 0.001 & $1.78(1.21-2.60)$ & 0.003 \\
\hline \multicolumn{5}{|l|}{ Augmentation } \\
\hline Infliximab & Reference & & Reference & \\
\hline Adalimumab & $1.01(0.84-1.22)$ & 0.903 & $1.13(0.92-1.37)$ & 0.238 \\
\hline Golimumab & $0.76(0.52-1.11)$ & 0.159 & $0.89(0.61-1.32)$ & 0.571 \\
\hline \multicolumn{5}{|l|}{ Dose escalation } \\
\hline Infliximab & Reference & & Reference & \\
\hline Adalimumab & $4.15(2.87-5.60)$ & $<0.001$ & $4.35(2.97-6.38)$ & $<0.001$ \\
\hline Golimumab & $1.27(0.57-2.84)$ & 0.558 & $1.33(0.59-3.02)$ & 0.494 \\
\hline \multicolumn{5}{|l|}{ Colectomy } \\
\hline Infliximab & Reference & & Reference & \\
\hline Adalimumab & $1.32(0.49-3.55)$ & 0.583 & $1.48(0.54-4.05)$ & 0.443 \\
\hline Golimumab & $0.84(0.11-6.72)$ & 0.873 & $0.80(0.10-6.60)$ & 0.832 \\
\hline
\end{tabular}

Cl, confidence interval.

*Hazard ratios estimated with multivariable analysis were adjusted for covariates such as sex, age, Charlson comorbidity index score, insurance type, type of medical facilities, concomitant treatments, and prior treatments.

benefits of adalimumab dose escalation or preferences for other biologic options might have been related with a lower rate of weekly dosing of adalimumab. ${ }^{25,26}$

There have been several previous studies comparing the risk of suboptimal responses according to biologic therapies for patients with UC. In a U.S. retrospective study using the Truven MarketScan database, the persistence rates of infliximab, adalimumab, and golimumab did not significantly differ from one another. ${ }^{20}$ In a recent nationwide Korean study comparing 630 patients prescribed infliximab and 232 patients prescribed adalimumab as the first anti-TNF agent for UC, there were no differences in colectomy rates, emergency room visits, hospitalization, and corticosteroid rescue. ${ }^{27}$ However, other outcomes, such as drug persistence and switching to other biologics, were not evaluated in that study. ${ }^{27}$ In another Korean study, UC patients treated with infliximab had a lower risk of drug discontinuation than those treated with golimumab, as well as a lower risk of switching than patients treated with adalimumab and golimumab. ${ }^{22}$ Reasons for the superiority of infliximab compared with other anti-TNF agents in terms of persistence and effectiveness have been hypothesized. Recent network meta-analyses on bio-naïve patients with UC have reported that infliximab was better than adalimumab for inducing clinical remission or responses and endoscopic improvement or mucosal healing, ${ }^{28-30}$ and infliximab was also superior to golimumab for inducing endoscopic improvement or mucosal healing. ${ }^{29,30}$ Regarding safety, a meta-analysis ${ }^{31}$ and a Danish nationwide study $^{32}$ demonstrated that infliximab-based therapy was associated with a lower risk of serious infections compared with adalimumab-based therapy among patients with UC. These results could partially explain the differences in suboptimal response frequencies between the three-drug groups in our study. However, because detailed reasons for discontinuing and switching of drugs cannot be evaluated from our study design, further research connecting claims data and patient-level data are required.

The major strength of our study is that we used the HIRA database based on a single-payer public medical insurance system in Korea combined with a rare/intractable diseases patient support program. Because almost all Korean patients are covered by the unified public medical insurance system provided by the Korean government, and the cost of UC management can be reimbursed with a $10 \%$ co-payment only after registration in the rare/intractable 
diseases patient support program, our findings could be generalized to the entire population of patients with UC receiving anti-TNF treatment in Korea. Secondly, because universal reimbursement criteria are applied for patients in Korea, UC patients in our study were likely to have received relatively uniform disease management. Thirdly, we comprehensively evaluated indicators of suboptimal responses to anti-TNF therapy for UC patients, including discontinuation, switching, augmentation of conventional therapy, dose escalation, and colectomy, and this is the first report of its kind to date at a nationwide population level. However, several limitations also should be noted. First of all, this study could not control all factors potentially affecting suboptimal events, since only the prescription information was available for analysis with the HIRA data. Even though characteristics of UC patients who received biologic therapies are expected to be similar when considering the universal reimbursement criteria for biologic therapy and baseline characteristics observed, other factors such as duration of UC, extent of UC, disease severity, adherence to drugs, anti-TNF serum levels, distinction between original and biosimilar infliximab, could not be captured because our study was limited by the information available in the database. These variables might have affected the suboptimal responses to anti-TNF agents. Similarly, detailed clinical and objective measures of treatment responses at an individual patient level were not available in the claims data. Through future studies such as the MOSAIK cohort study, which is a prospective cohort study that collects detailed characteristics of patients and disease including UC extent and severity to search for disease course, treatment response, and prognostic factors, the limitations of the current study based on the claims database could be overcome and more information on the real-life effectiveness of biologics for UC patients could be provided. ${ }^{33}$ Additionally, non-reimbursed healthcare resources were not available in the HIRA database and not identified in this study. However, due to the high cost of anti-TNF agents, there would have been virtually no cases of self-paid treatment with anti-TNFs. Next, the study medications were included only after reimbursement during the study period. There were fewer patients in the golimumab cohort, and patients treated with golimumab had a shorter follow-up duration than those in the infliximab and adalimumab cohorts, due to late reimbursement in Korea (infliximab in October 2010, adalimumab in July 2013 and golimumab in April 2015). Thus, the follow-up durations for patients in the golimumab cohort were inevitably limited. Therefore, if the effectiveness of golimumab is investigated with a sufficient sample size for a longer follow-up period, the results could be different from those of the current study. Additionally, because vedolizumab was reimbursed in August 2017, it was not included as an index treatment in the present study. Lastly, caution should be taken when applying our results to other patient populations, considering differences in medical care systems, genetics, and disease characteristics.

In conclusion, this nationwide population-based study showed the real-life incidences of suboptimal responses to anti-TNF therapy and their detailed features. These findings could be helpful for estimating the effectiveness and safety of anti-TNF therapies and also for supporting decision-making regarding treatment strategies for UC patients in the real-world.

\section{CONFLICTS OF INTEREST}

B.D.Y. has received a research grant from Celltrion and Pfizer Korea; consulting fees from Abbvie Korea, Celltrion, Chong Kun Dang Pharm., Daewoong Pharma., Ferring Korea, Janssen Korea, Kangstem Biotech, LG Chem., Medtronic Korea, Pfizer Korea, Shire Korea, Takeda Korea, IQVIA, Cornerstones Health, and Takeda; speaking fees from Abbvie Korea, Celltrion, Ferring Korea, Janssen Korea, Pfizer Korea, Shire Korea, Takeda Korea, and IQVIA. J.Y.S. has received research funding from the Ministry of Food and Drug Safety, the Ministry of Health and Welfare, the National Research Foundation, and the Governmentwide R\&D Fund for Infectious Disease Research in Korea, Amgen Korea, Pfizer Korea, Hoffmann-La Roche Korea and Yungjin Korea. H.M.P. and M.Y.L. are employees of VIAplus. J.Y.J. and H.J.Y. Yoo are employees and shareholders of Pfizer Inc. Except for that, no potential conflict of interest relevant to this article was reported.

\section{ACKNOWLEDGEMENTS}

This study was sponsored by Pfizer Pharmaceuticals Korea Ltd.

\section{AUTHOR CONTRIBUTIONS}

Study design: J.Y.S., H.M.P., M.Y.L., J.Y.J., H.J.Y., B.D.Y. Statistical analysis: H.M.P., B.D.Y. Writing of the draft manuscript: J.Y.S., H.M.P. Critical revision of the manuscript: J.Y.S., H.M.P., M.Y.L., J.Y.J., H.J.Y., B.D.Y. Study supervision: B.D.Y. 


\section{ORICD}

Ju-Young Shin https://orcid.org/0000-0003-1010-7525

Hye-Min Park https://orcid.org/0000-0003-0818-9410

Min-Young Lee https://orcid.org/0000-0003-1649-3025

Ja-Young Jeon https://orcid.org/0000-0002-1930-6857

Hyun-Jeong Yoo https://orcid.org/0000-0002-7514-7432

Byong Duk Ye https://orcid.org/0000-0001-6647-6325

\section{REFERENCES}

1. Magro F, Gionchetti P, Eliakim R, et al. Third European evidence-based consensus on diagnosis and management of ulcerative colitis. Part 1: definitions, diagnosis, extra-intestinal manifestations, pregnancy, cancer surveillance, surgery, and ileo-anal pouch disorders. J Crohns Colitis 2017;11:649-670.

2. Choi CH, Moon W, Kim YS, et al. Second Korean guideline for the management of ulcerative colitis. Korean J Gastroenterol 2017;69:1-28.

3. Ng WK, Wong SH, Ng SC. Changing epidemiological trends of inflammatory bowel disease in Asia. Intest Res 2016;14: 111-119.

4. Kaplan GG, Ng SC. Understanding and preventing the global increase of inflammatory bowel disease. Gastroenterology 2017;152:313-321.

5. Kotze PG, Underwood FE, Damião AOMC, et al. Progression of inflammatory bowel diseases throughout Latin America and the Caribbean: a systematic review. Clin Gastroenterol Hepatol 2020;18:304-312.

6. Park SH, Kim YJ, Rhee KH, et al. A 30-year trend analysis in the epidemiology of inflammatory bowel disease in the Songpa-Kangdong District of Seoul, Korea in 1986-2015. J Crohns Colitis 2019;13:1410-1417.

7. Kwak MS, Cha JM, Lee HH, et al. Emerging trends of inflammatory bowel disease in South Korea: a nationwide population-based study. J Gastroenterol Hepatol 2019;34:10181026.

8. Harbord M, Eliakim R, Bettenworth D, et al. Third European evidence-based consensus on diagnosis and management of ulcerative colitis. Part 2: current management. J Crohns Colitis 2017;11:769-784.

9. Xu J, Tang M, Shen J. Trends and factors affecting hospitalization costs in patients with inflammatory bowel disease: a two-center study over the past decade. Gastroenterol Res Pract 2013;2013:267630.

10. van der Valk ME, Mangen MJ, Leenders M, et al. Healthcare costs of inflammatory bowel disease have shifted from hospitalisation and surgery towards anti-TNFa therapy: results from the COIN study. Gut 2014;63:72-79.

11. Kim JW, Lee CK, Lee JK, et al. Long-term evolution of direct healthcare costs for inflammatory bowel diseases: a population-based study (2006-2015). Scand J Gastroenterol 2019; 54:419-426.

12. Saito S, Nakazawa K, Suzuki K, Ishikawa T, Akazawa K. Paradigm shift of healthcare cost for patients with inflammatory bowel diseases: a claims data-based analysis in Japan. Gastrointest Disord 2019;1:120-128.

13. Lee J, Im JP, Han K, et al. Changes in direct healthcare costs before and after the diagnosis of inflammatory bowel disease: a nationwide population-based study. Gut Liver 2020; 14:89-99.

14. Pugliese D, Felice C, Papa A, et al. Anti TNF- $\alpha$ therapy for ulcerative colitis: current status and prospects for the future. Expert Rev Clin Immunol 2017;13:223-233.

15. Bonovas S, Pantavou K, Evripidou D, et al. Safety of biological therapies in ulcerative colitis: an umbrella review of meta-analyses. Best Pract Res Clin Gastroenterol 2018;3233:43-47.

16. Rubin DT, Mody R, Davis KL, Wang CC. Real-world assessment of therapy changes, suboptimal treatment and associated costs in patients with ulcerative colitis or Crohn's disease. Aliment Pharmacol Ther 2014;39:1143-1155.

17. Patel H, Lissoos T, Rubin DT. Indicators of suboptimal biologic therapy over time in patients with ulcerative colitis and Crohn's disease in the United States. PLoS One 2017;12: e0175099.

18. Lindsay JO, Armuzzi A, Gisbert JP, et al. Indicators of suboptimal tumor necrosis factor antagonist therapy in inflammatory bowel disease. Dig Liver Dis 2017;49:1086-1091.

19. Gemayel NC, Rizzello E, Atanasov P, Wirth D, Borsi A. Dose escalation and switching of biologics in ulcerative colitis: a systematic literature review in real-world evidence. Curr Med Res Opin 2019;35:1911-1923.

20. Chen C, Hartzema AG, Xiao H, et al. Real-world pattern of biologic use in patients with inflammatory bowel disease: treatment persistence, switching, and importance of concurrent immunosuppressive therapy. Inflamm Bowel Dis 2019; 25:1417-1427.

21. Jang EJ, Ha JE, Im SG, Kim MG, Sohn HS. A real-world analysis of prescribing patterns and non-persistence of antiTNF $\alpha$ therapy for inflammatory bowel disease. Clin Drug Investig 2019;39:625-630.

22. Jung YS, Han M, Park S, Cheon JH. Biologic use patterns and predictors for non-persistence and switching of biologics in patients with inflammatory bowel disease: a nationwide population-based study. Dig Dis Sci 2020;65:1436-1444.

23. Kim JA, Yoon S, Kim LY, Kim DS. Towards actualizing the value potential of Korea Health Insurance Review and Assessment (HIRA) data as a resource for health research: strengths, limitations, applications, and strategies for optimal use of HIRA data. J Korean Med Sci 2017;32:718-728. 
24. Kim KH. Comorbidity adjustment in health insurance claim database. Health Policy Manag 2016;26:71-78.

25. Wolf D, D’Haens G, Sandborn WJ, et al. Escalation to weekly dosing recaptures response in adalimumab-treated patients with moderately to severely active ulcerative colitis. Aliment Pharmacol Ther 2014;40:486-497.

26. Colombel JF, Sandborn WJ, Ghosh S, et al. Four-year maintenance treatment with adalimumab in patients with moderately to severely active ulcerative colitis: data from ULTRA 1, 2, and 3. Am J Gastroenterol 2014;109:1771-1780.

27. Han M, Jung YS, Cheon JH, Park S. Comparison of realworld outcomes of infliximab versus adalimumab in biologic-naïve Korean patients with ulcerative colitis: a populationbased study. Yonsei Med J 2020;61:48-55.

28. Vickers AD, Ainsworth C, Mody R, et al. Systematic review with network meta-analysis: comparative efficacy of biologics in the treatment of moderately to severely active ulcerative colitis. PLoS One 2016;11:e0165435.

29. Singh S, Fumery M, Sandborn WJ, Murad MH. Systematic review with network meta-analysis: first- and second-line pharmacotherapy for moderate-severe ulcerative colitis. Aliment Pharmacol Ther 2018;47:162-175.

30. Singh S, Murad MH, Fumery M, Dulai PS, Sandborn WJ. First- and second-line pharmacotherapies for patients with moderate to severely active ulcerative colitis: an updated network meta-analysis. Clin Gastroenterol Hepatol 2020;18: 2179-2191.

31. Singh S, Facciorusso A, Dulai PS, Jairath V, Sandborn WJ. comparative risk of serious infections with biologic and/or immunosuppressive therapy in patients with inflammatory bowel diseases: a systematic review and meta-analysis. Clin Gastroenterol Hepatol 2020;18:69-81.

32. Singh S, Andersen NN, Andersson M, Loftus EV Jr, Jess T. Comparison of infliximab and adalimumab in biologicnaive patients with ulcerative colitis: a nationwide Danish cohort study. Clin Gastroenterol Hepatol 2017;15:1218-1225.

33. Lee CK, Lee KM, Park DI, et al. A new opportunity for innovative inflammatory bowel disease research: the moderateto-severe ulcerative colitis in Korea (MOSAIK) cohort study. Intest Res 2019;17:1-5. 